\title{
ANÁLISE DE MATERIAL DIDÁTICO PARA A PRODUÇÃO DE ENSAIO ACADÊMICO NO CURSO DE LETRAS FRANCÊS-PORTUGUÊS
}

\section{ANALYSIS OF TEACHING MATERIAL FOR THE PRODUCTION OF ACADEMIC TESTS IN THE FRENCH-PORTUGUESE LETTER COURSE}

Emerson Patrício de Morais Filho*

Josilene Pinheiro-Mariz**

Resumo: Este artigo tem por objetivo analisar o material didático utilizado para o ensino de produção de ensaio acadêmico em língua francesa, no curso de Letras português-francês, de uma universidade brasileira. Assim, buscamos identificar a abordagem de ensino de escrita subjacente à proposta do material. Para a análise, nos baseamos no conceito de letramentos trazido por Kleiman (1995) e nos modelos de abordagens da escrita e do letramento do estudante em contextos acadêmicos, descritos por Lea e Street (1998). Nossa pesquisa é de tipo documental. Os resultados demonstram que o material analisado se insere no modelo de habilidades de estudo, isto é, a proposta didática sugere uma abordagem genérica de ensino de produção do gênero ensaio, levando-se em conta apenas os seus aspectos linguísticos, deixando de lado as especificidades disciplinares para a produção do gênero em questão.

Palavras-chave: Ensaio; escrita; francês língua estrangeira.

Aвstract: This article aims to analyze the didactic material used to teach academic essay production in French, in the course of Portuguese-French Letters, from a Brazilian university. Thus, we aim to identify the writing teaching approach that underlies the material proposal. For the analysis, we are based on the concept of literacies brought by Kleiman (1995) and on the models of approaches to writing and literacy of students in academic contexts, described by Lea and Street (1998). Our research is of documentary type. The results show that the analyzed material is part of the study skills model, that is, the didactic proposal suggests a generic approach to teaching production of the

\footnotetext{
"Doutorando em Linguagem e Ensino pelo PPGLE - UFCG. E-mail: epmf.fr@hotmail.com.

"Doutora em Letras pela Universidade de São Paulo (USP). Professora associada da Universidade Federal de Campina Grande (UFCG). Docente do PPGLE - UFCG. E-mail: jsmariz22@hotmail.com.
} 
essay genre, taking into account only its linguistic aspects, without considering the disciplinary specificities for the production of the genre in question.

KEYwords: Essay; writing; French as a foreign language.

\section{INTRODUÇÃo}

A produção de gêneros acadêmicos é uma atividade muito complexa, pois demanda, daqueles que a praticam, habilidades múltiplas, que vão muito além de conhecimentos linguísticos (correção gramatical, coesão, coerência etc.) e estruturais dos gêneros produzidos nesse meio. Os gêneros textuais, por extensão os gêneros acadêmicos, são práticas sociais que estão fortemente imbricadas ao contexto em que são produzidos. Portanto, cada comunidade discursiva tem suas próprias normas e convenções para a construção do conhecimento e para a produção dos gêneros que circulam na comunidade acadêmica. Além disso, os textos variam linguisticamente de acordo com sua finalidade e contexto de produção. Assim sendo, um bom domínio linguístico e estrutural não garante uma produção textual bem-sucedida e aceita em uma dada comunidade discursiva, pois há níveis mais profundos de competências que subjazem a essas produções que se encontram no viés epistemológico. Acrescente-se a isso, a dificuldade de se produzir gêneros acadêmicos em língua estrangeira (doravante LE), como no contexto dos cursos de formação de professores de LE, no caso específico, professores de francês. O desafio do professor formador se torna ainda maior, pois, muitas vezes, os estudantes apresentam ainda dificuldades no domínio da LE.

Nesse contexto, no presente artigo, buscamos analisar um material didático utilizado na disciplina de "Práticas de Leitura e Escrita de Textos Acadêmicos em Língua Francesa" para a produção de ensaio acadêmico em língua francesa, do curso de licenciatura em Letras português-francês da Universidade Federal de Campina Grande (UFCG). A disciplina é de caráter obrigatório e é ofertada no sexto período do curso, ou seja, no penúltimo semestre, quando os alunos já estão se preparando para escrever os trabalhos de conclusão de curso (TCC). O objetivo da disciplina é o de habilitar os alunos a produzirem gêneros acadêmicos variados em língua francesa, tais como: relatório, resumo, fichamento, artigo científico, ensaio acadêmico etc. O material que é objeto de nossa análise neste trabalho é um documento que tem por finalidade capacitar os estudantes a produzirem um ensaio acadêmico em língua francesa. $\mathrm{O}$ documento intitulado "Écrire un essai"/ "escrever um ensaio" (anexo I) é uma ficha pedagógica destinada ao uso de professores e estudantes de francês língua estrangeira (FLE). Esse material foi produzido e editado em 2014 e modificado em 2016, pela autora Sophie Garcia. A autora tem um $b \log ^{1}$ voltado ao ensino-aprendizagem de língua francesa, no qual ela disponibiliza

${ }^{1}$ Endereço eletrônico: www.agirenfrancais.com. Acesso em: 14 dez. 2020. 
vários materiais didáticos e de onde foi retirado o material desta análise. Portanto, nosso interesse nesta investigação consiste em identificar a abordagem de ensino de escrita que está subjacente à proposta desse material para a produção do gênero ensaio acadêmico. Como objetivos específicos, buscamos identificar as vantagens e limitações da proposta do material e discutir a necessidade de elaboração de material didático, que promova o letramento dos alunos, em contexto universitário, de maneira plena e eficaz.

A escolha do gênero ensaio, em específico, se deu pela complexidade desse gênero, no que tange à estrutura, ao domínio dos conteúdos disciplinares, ao campo epistemológico e, sobretudo, no que concerne ao desafio de ensiná-lo a estudantes que ainda apresentam dificuldades com o domínio da língua francesa.

Na próxima seção, descrevemos os fundamentos teórico-metodológicos que norteiam nossa pesquisa.

\section{FundAMENTAÇÃo TEÓRICA}

Dada a complexidade das práticas de letramentos acadêmicos, desde a década de 1980, muitos pesquisadores têm se interessado em compreender as dimensões subjetivas dessas práticas e sistematizar seu ensino e aprendizagem. Dessa forma, algumas teorias recentes têm se destacado nessas pesquisas - como: escrita no âmbito das disciplinas, estudos dos gêneros e letramentos acadêmicos -, no intuito de discutir a possibilidade e a maneira de adaptar a teoria à prática. Em cada uma dessas teorias, abordagens distintas são propostas a fim de promover o letramento dos estudantes. Em nossa pesquisa, adotamos o conceito de letramentos (no plural) trazido por Kleiman (1995, p. 16), como sendo práticas sociais e culturais dos diversos grupos que envolvem o uso da escrita, tanto em recepção quanto em produção. Empregamos o termo no plural, por entendermos que essas práticas são múltiplas e variadas, de acordo com o contexto sociocomunicativo e, portanto, demandam habilidades e conhecimentos específicos. Lea e Street (1998) descrevem três modelos de abordagens da escrita e do letramento do estudante em contextos acadêmicos, são eles: (i) modelo de habilidades de estudo; (ii) modelo de socialização acadêmica; e (iii) modelo de letramentos acadêmicos.

O primeiro modelo, o das habilidades de estudo, segundo os autores, concebe a escrita e o letramento como habilidade individual e cognitiva, na qual os aspectos superficiais da forma da língua são o foco (LEA; STREET, 2014, p. 479). Nessa abordagem, pressupõe-se que o domínio das regras gramaticais e da sintaxe, além do domínio das regras de pontuação e de ortografia, garante o êxito dos alunos nas práticas de letramento. Assim, os conhecimentos de escrita e de letramento são transferíveis para qualquer contexto, pois são formas padronizadas, que, uma vez apreendidas, podem ser aplicadas em qualquer contexto de produção.

O segundo modelo, denominado socialização acadêmica, é descrito pelos autores como uma abordagem que preconiza a aculturação dos estudantes quanto a discursos e a gêneros 
baseados em temas e em disciplinas (LEA; STREET, 2014, p. 479). Essa abordagem pressupõe que a imersão do aluno em práticas de letramentos situadas faz com que ele adquira, de forma natural, os modos de falar, de escrever, de pensar e de interagir, que caracterizam membros de comunidade disciplinar ou temática. Esse modelo parte do pressuposto que os discursos disciplinares e os gêneros são relativamente estáveis e que, tendo os estudantes dominado e entendido as regras básicas de um discurso acadêmico particular, estariam aptos a reproduzi-lo sem problemas (LEA; STREET, 2014, p. 479).

O terceiro modelo, o de letramentos acadêmicos, está mais interessado nas dimensões de produção de sentido, identidade, poder e autoridade das práticas letradas, sem, contudo, negligenciar as outras dimensões. Esse modelo coloca em primeiro plano a natureza institucional daquilo que conta como conhecimento em qualquer contexto acadêmico específico (LEA; STREET, 2014, p. 479). Os autores ressaltam que o modelo de letramentos acadêmicos se assemelha, em muitos aspectos, ao modelo de socialização acadêmica, salvo pelo fato de considerar os processos envolvidos na aquisição de usos adequados e eficazes de letramento como mais complexos, dinâmicos, matizados e situados, o que abrange tanto questões epistemológicas quanto processos sociais incluindo: relações de poder entre pessoas, instituições e identidades sociais (LEA; STREET, 2014, p. 479).

Vale salientar que esses modelos não são mutuamente exclusivos, mas complementares, isto é, cada um engloba o anterior e o amplia. Portanto, o modelo de letramentos acadêmicos engloba várias características do modelo de habilidades de estudo e do modelo de socialização acadêmica. No entanto, o modelo de letramentos acadêmicos, segundo Lea e Street (1998), é o que melhor leva em conta a natureza da produção textual do aluno em relação às práticas institucionais, relações de poder e identidades. Em resumo, esse modelo consegue contemplar a complexidade da construção de sentidos, ao contrário dos outros dois modelos (LEA; STREET, 2014, p. 479).

No âmbito acadêmico, apesar de na teoria já se ter avançado bastante em relação às abordagens de ensino de produção textual, na prática, esses avanços parecem ainda não ter acompanhado o mesmo ritmo da teoria. Na academia, o modelo que predomina ainda hoje é o das habilidades de estudo, que tende "a enfatizar o uso de listas padronizadas de itens a serem seguidos, geralmente em relação à estrutura do texto acadêmico (por exemplo: introdução, referencial teórico, métodos e dados)" (STREET, 2010, p. 542). Assim sendo, buscamos problematizar a necessidade de se desenvolverem abordagens que contemplem a complexidade do ensino da produção escrita dos gêneros acadêmicos em detrimento de abordagens genéricas, que abordam esses gêneros de forma simplista e superficial.

Na próxima seção, apresentamos o gênero ensaio acadêmico e, em seguida, procedemos à nossa análise. 


\section{O GÊNERO ENSAIO ACADÊMICO}

No âmbito acadêmico, são muitos os gêneros que circulam: artigo científico, ensaio, relatório, resenha, resumo, fichamento, projeto de pesquisa, dissertação, tese etc. A apropriação desses gêneros, tanto em recepção como em produção, por parte dos alunos, é de fundamental importância. No entanto, muitas vezes, não há disciplinas específicas nos cursos voltadas para o ensino desses gêneros na academia. Há, ainda hoje, uma crença de que um bom domínio linguístico (de regras gramaticais, de coesão e de coerência) garante boas produções desses gêneros e, portanto, as dificuldades dos alunos são tratadas como déficits de natureza linguística. Entretanto, quando observamos mais de perto a composição desses gêneros, percebemos que suas produções implicam muito mais do que apenas um bom domínio linguístico. Dessa forma, torna-se necessário uma abordagem desses gêneros que leve em conta as especificidades disciplinares da comunidade discursiva na qual são produzidos.

Dentre os gêneros que circulam no meio acadêmico, o ensaio é um gênero que apresenta certa dificuldade por parte dos alunos, pela complexidade da sua composição. Muitas vezes, não fica muito claro para os alunos os elementos de composição escrita desse gênero. Portanto, faz-se necessário um ensino sistemático e aprofundado dos seus elementos constitutivos e dos critérios avaliativos adotados pelos professores para a correção dessas produções.

Segundo Campos (2015), o gênero ensaio tem o objetivo de analisar um fato específico e de apresentar uma reflexão e um posicionamento sobre tal fato. Esse gênero se diferencia do artigo científico, segundo a autora, pelo fato de ser mais sintético e subjetivo do que este. Entretanto, a redação desse gênero deve ser bem fundamentada na área ou no domínio discursivo ao qual está vinculado (CAMPOS, 2015, p. 4). Campos (2015) acrescenta que, muitas vezes, há certa confusão no meio acadêmico em relação à diferença entre o ensaio acadêmico e o artigo científico, sendo distinguidos apenas pela sua extensão: o artigo científico é considerado mais extenso.

O ensaio é um gênero de muita importância no âmbito acadêmico, pois ele permite uma "contribuição interpretativa original de casos, dados e conceitos de domínio de uma área específica do conhecimento e que podem ajudar as demais pessoas, pertencentes ou não àquela comunidade discursiva, a refletirem sobre determinada questão, muitas vezes, polêmica" (CAMPOS, 2015, p. 5). Portanto, a produção desse gênero possibilita "um processo significativo de construção do conhecimento e não apenas de reprodução do conhecimento, já que possibilita reflexão e avaliação crítica do objeto-caso dado a estudo" (CAMPOS, 2015, p. 5). Por ser um gênero que permite maior subjetividade e liberdade de "expressão" autoral, os ensaios acadêmicos costumam ser escritos em primeira pessoa do singular (CAMPOS, 2015, p. 6). A estrutura desse gênero é constituída, segundo Campos (2015), por: título, autor e afiliação, resumo, introdução, desenvolvimento, considerações finais e referências. 
Na próxima seção, descrevemos e analisamos a composição do material e a proposta sugerida.

\section{AnÁLISE}

O documento em questão foi editado em arquivo PDF e busca orientar, de forma sucinta, a produção de um ensaio. O material sistematiza a proposta em três etapas: (i) antes da escrita; (ii) a escrita; e (iii) a releitura. O texto é iniciado com uma definição do gênero e das suas partes constitutivas. Para a autora, escrever um ensaio é, antes de tudo, responder a uma questão e exprimir sua opinião, argumentando. A autora divide a composição do gênero ensaio em três grandes partes: (i) a introdução; (ii) o desenvolvimento; e (iii) a conclusão.

Na primeira parte, intitulada "antes da redação", Garcia (2016 [2014]) orienta que a escolha do tema do ensaio deve ser pautada numa questão que não coloque o aluno em dificuldade. Ela sugere que se coloque, de preferência, uma questão fechada em vez de uma questão aberta. A título de exemplo, a autora propõe as seguintes problemáticas para a produção de um ensaio: deve-se autorizar a televisão às crianças com menos de três anos? É preciso proibir o uso de cigarro? Como evitar que os adolescentes bebam muito álcool na noitada? etc. Após a escolha da problemática, a autora orienta que se elabore os planos argumentativos para a composição do texto, que podem estar divididos, segundo a problemática, em: sim ou não; a favor ou contra; tema 1 , tema 2 , tema 3 etc. A última fase dessa etapa consiste em procurar os argumentos e exemplos que servirão na redação do ensaio.

Percebe-se, desde o início, que a proposta da autora para a construção de um ensaio está pautada em um modelo que se preocupa muito mais com a forma do que com o conteúdo a ser tratado. Assim, a proposta de escrita busca focar temas gerais do cotidiano que não necessitam de um conhecimento específico aprofundado do tema a ser tratado. Esse modelo pressupõe que os domínios das regras gramaticais, da sintaxe, do domínio das regras de pontuação e da ortografia garantem o êxito dos alunos nas práticas de letramentos. Além disso, há uma ideia subjacente neste tipo de proposta de que os gêneros são formas padronizadas e que independem do meio em que são produzidos.

Na segunda parte da proposta, intitulada "a redação", Garcia (2016 [2014]) sugere que a composição do ensaio seja dividida em, basicamente, três partes: (i) a introdução; (ii) o desenvolvimento (parte 1, parte 2); e (iii) a conclusão. A introdução, para a autora, deve ser clara e simples, e, nela, o autor deve colocar as bases para o desenvolvimento sem deixar aparecer sua opinião. Ela é composta, segundo Garcia (2016 [2014]), por três partes: (i) apresentação do tema no seu contexto; (ii) a problemática escolhida para a reflexão; e (iii) o anúncio do plano textual.

Para a parte do desenvolvimento, a autora orienta que se escolha, mais ou menos, o mesmo número de argumentos para cada parte, a fim de que o texto fique equilibrado. Para que o desenvolvimento fique bem organizado e bem articulado, a autora apresenta uma lista 
de marcadores lógicos (conjunções e advérbios) para relacionar as diferentes frases: advérbios de tempo, conjunções explicativas, concessivas, causais, alternativas, conclusivas etc. Em seguida, a autora apresenta algumas expressões para se introduzir a opinião do autor, como: "eu acho que”, “eu penso que”, "a meu ver”, “em minha opinião” etc.

Para a redação da conclusão, Garcia (2016 [2014]) sugere que essa seção seja composta de três partes: resposta da problemática, a opinião do autor e a abertura do tema. Na resposta da problemática, a autora orienta que se faça uma síntese dos pontos importantes citados ao longo do texto. A opinião do autor, segundo a autora, pode ser feita de forma modalizada, sem necessariamente uma tomada de posição incisiva. Na última parte, na abertura do tema, a autora orienta que o autor pode direcionar o tema para outro caminho.

Finalmente, na última seção, intitulada "a releitura”, Garcia (2016 [2014]) dá alguns conselhos para a verificação da coerência do ensaio. Assim, a autora orienta que se observe se as ideias no texto estão encadeadas de forma lógica, que se observe se há repetições de palavras ou de ideias, e que se verifique também se as ideias apresentadas não se contradizem. Além disso, Garcia (2016 [2014]) chama a atenção para que não se utilizem frases prontas e que se evite a neutralidade no texto.

Percebemos, após essa descrição, que a proposta apresentada pela autora é bem sistematizada e organizada. No entanto, ela apresenta algumas limitações. Primeiramente, ao se preconizar o ensino explícito de aspectos linguísticos do gênero ensaio, por meio de uma abordagem genérica, desconsidera-se o fato de que os gêneros são práticas sociais fortemente vinculadas ao contexto de produção e que cada comunidade discursiva tem suas próprias normas e convenções para a construção do conhecimento e a produção dos gêneros que circulam na comunidade acadêmica. Portanto, o mesmo gênero pode ser concebido de maneiras distintas em campos diferentes de produção sociocomunicativa. Além disso, os textos variam linguisticamente de acordo com sua finalidade e com seu contexto de produção. Assim, uma abordagem reducionista, que desconsidera essas variáveis como estratégia para facilitar o trabalho do professor, desemboca, inexoravelmente, em um ensino da escrita incompleto e deficiente, não respondendo às necessidades reais dos estudantes.

Outro fato que também fica visível nessa abordagem é o papel intervencionista desempenhado pelo professor. Sua função nessa abordagem é, antes de tudo, o de prescrever as orientações para a produção do gênero e regular/corrigir a produção dos alunos. Os alunos, nesse contexto, exercem uma função passiva de apenas receber as orientações do professor e executá-las, não havendo nenhum trabalho de reflexão anterior à produção sobre a composição, a função e os objetivos desse gênero.

Percebe-se também que toda a atenção da proposta se volta para aspectos linguísticos (utilização de marcadores lógico-argumentativos) e regras de coesão e coerência textual. Portanto, a proposta se insere numa abordagem de ensino de escrita que Lea e Street (1998, p. 162) qualificam de "modelo de estudo das habilidades cognitivas", pois desconsideram 
os modos de falar, de escrever, de pensar e de interagir, que caracterizam membros de comunidade disciplinar e também as dimensões de produção de sentido, identidade, poder e autoridade das práticas letradas. Assim sendo, a abordagem apresenta muitas limitações no que concerne ao letramento pleno dos estudantes.

Por outro lado, uma abordagem genérica para o ensino da escrita acadêmica, sem se levar em conta as especificidades epistemológicas das diversas áreas de produção sociocomunicativa, é uma prática bastante criticada pela teoria dos letramentos acadêmicos. Esse tipo de abordagem se baseia no pressuposto de que existem aspectos comuns na escrita acadêmica que podem ser ensinados aos alunos, independentemente da disciplina que estejam cursando. Esse modelo de ensino, chamado por Lea e Street (1998, p. 162) de "modelo de estudo das habilidades cognitivas" é criticado por diversas razões, a saber:

- trata apenas de aspectos linguísticos superficiais;

- transfere dos professores/tutores da disciplina (que definem e avaliam as tarefas de produção textual) para agentes externos à disciplina a responsabilidade de apoiar os alunos; e

- negligencia a relação entre a escrita e a construção do conhecimento na disciplina (MITCHELL, 2006 apud STREET, 2010, p. 547).

Nesse sentido, o autor defende que o ensino da escrita no âmbito acadêmico seja feito a partir das especificidades das disciplinas nas quais são produzidas, pois não se podem deixar de lado os contextos sociocomunicativos, nos quais essas produções são realizadas, e as especificidades de cada área disciplinar.

Na próxima seção, apresentamos nossas conclusões e problematizamos a necessidade de se desenvolver materiais didáticos mais alinhados com a proposta dos letramentos acadêmicos, a fim de promover o letramento pleno e eficaz dos alunos para a leitura e a produção dos diversos gêneros que circulam na academia.

\section{À GUISA DE CONCLUSÃo}

Considerando as orientações presentes no modelo de letramentos acadêmicos (LEA; STREET, 1998), nossa análise evidencia a insuficiência do material didático em questão, no que tange ao pleno desenvolvimento do gênero ensaio nos aspectos estruturais e linguísticodiscursivos. A partir dos dados analisados, fica claro que uma abordagem genérica de ensino de produção de gêneros acadêmicos, levando-se em conta apenas os seus aspectos linguísticos superficiais, de correção gramatical, de coerência e de coesão textual, não são suficientes para habilitar os alunos nas produções desses gêneros em disciplinas específicas, pois cada comunidade discursiva tem suas próprias normas e convenções para a construção do conhecimento e para a produção dos gêneros que circulam na comunidade acadêmica. 
Além disso, os textos variam linguisticamente de acordo com sua finalidade e contexto de produção. Assim sendo, um bom domínio linguístico e estrutural não garante uma produção textual bem-sucedida e validada em uma dada comunidade discursiva, pois há níveis mais profundos que subjazem a essas produções que se encontram no viés epistemológico. Assim sendo, deve-se adotar uma abordagem que contemple essas dimensões, que estão no nível das práticas institucionais, das relações de poder, de identidades e da construção de sentidos. Nesse sentido, a perspectiva dos letramentos acadêmicos oferece uma rica reflexão e um referencial teórico que podem contribuir para orientar as práticas docentes no que se refere ao ensino de escrita em contexto acadêmico.

\section{REFERÊNCIAS}

CAMPOS, M. Manual de redação científica: ensaio acadêmico, relatório de experimento e artigo científico. Mariana: Edição do autor, 2015.

GARCIA, S. Écrire un essai. (2016 [2014]). Disponível em: www.agirenfrancais.com. Acesso em: 20 jun. 2019.

KLEIMAN, A. B. (Org.). Significados do letramento: uma nova perspectiva sobre a prática social da escrita. Campinas, São Paulo: Mercado de Letras, 1995.

LEA, M. R.; STREET, B. Student writing in higher education: an academic literacies approach. Studies in Higher Education, v. 23, n. 2, p. 157-172, jun. 1998.

LEA, M. R.; STREET, B. A escolarização do letramento. In: Letramentos sociais: abordagens críticas do letramento no desenvolvimento, na etnografia e na educação. Tradução de Marcos Bagno. São Paulo: Parábola, 2014. p. 121-144.

STREET, B. Os novos Estudos sobre o Letramento: histórico e perspectivas. In: MARINHO, M.; CARVALHO, G. (Ed.). Cultura escrita e Letramento. Belo Horizonte: UFMG, 2010. p. 33-53.

\section{ANEXo I - CORPUS DA ANÁLISE (GARCiA, 2016 [2014])}

\section{ÉCRIRE UN ESSAI}

Écrire un essai c'est avant tout répondre à une question et exprimer son opinion en argumentant et en donnant des exemples.

Il y a trois grandes parties dans un essai : L'introduction - Le développement - La conclusion

\section{Avant la rédaction}

$X$ Dans le cas où vous devez dégager la problématique d'un texte ou d'une image, choisissez une question qui ne vous mettra pas en difficulté. 
Il est souvent plus facile de répondre à une question fermée qu'à une question ouverte. Du moins, les plans seront différents.

Reliez les problématiques aux plans correspondants.

\section{Problématiques}

Doit-on autoriser la télévision aux enfants de moins de trois ans?

Pourquoi n'y a-t-il pas plus d'écoles qui imposent l'uniforme?

Faut-il interdire la e-cigarette?

Peut-on toujours choisir notre profession?

La cigarette est-elle dangereuse?

Comment éviter que les adolescents boivent trop d'alcool en soirée?

\section{Plans}

OUI - NON POUR - CONTRE

Thème 1 - Thème 2 (- Thème 3 )

XUne fois la problématique et le type de plan définis, vous devez chercher vos arguments et vos exemples.

X Les exemples pourront être pris dans votre quotidien, vos expériences personnelles, votre culture générale, ....

X Les exemples peuvent être illustratifs, c'est-à-dire placés après l'argument ou bien argumentatifs, c'est-à-dire placés avant ou se superposant à l'argument.

$X$ Si vous ne pouvez pas expliquer clairement votre argument, c'est qu'il n'est pas bon.

\begin{tabular}{c|c|c|c}
\hline & Idées essentielles & Arguments & Exemples \\
\hline Première Partie & OUI & 1. & 1. \\
\hline & POUR & 2. & 2. \\
\hline Deuxième Partie & THËME I & 3. & 3. \\
\hline & NON & 1. & 1. \\
\hline & CONTRE & 2. & 2. \\
\hline (Troisième Partie) & THËME 2 & 3. & 3. \\
\hline & & 1. & 1. \\
\hline & & 2. & 2. \\
\hline
\end{tabular}




\section{LA RÉDACTION}

L'organisation de votre essai doit être très claire. Il s'agit de voir les différentes parties de l'essai avant même de le lire.

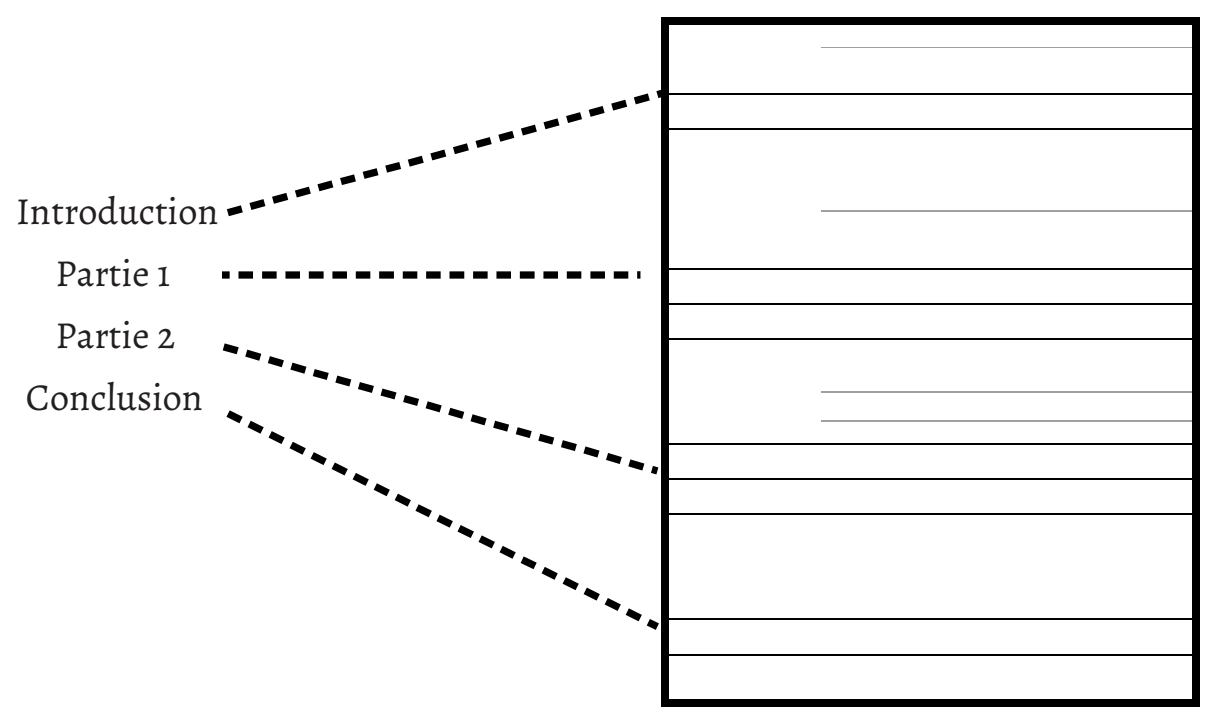

XÉcrire l'introduction. Celle-ci doit être simple et claire. Il s'agit des premières phrases lues par les lecteurs, vous devez donc bien poser les bases de votre développement sans laisser apparaître votre opinion. Elle est composée de trois parties.

1. Amener le sujet $=$ Une phrase présentant le thème dans son contexte.

2. La problématique = La question que vous avez choisie lors de votre réflexion. Vous pouvez poser directement la question ou bien utiliser le discours indirect : Nous pouvons alors nous demander si/pourquoi/comment...

3. Annoncer le plan = Indiquer le fil de votre pensée aux lecteurs afin qu'ils suivent votre développement. Il est préférable - car moins scolaire - d'annoncer le plan de manière indirecte, en posant des questions, en articulant une phrase de façon que le plan s'en dégage. Néanmoins, si cela vous semble trop compliqué, écrivez plusieurs phrases présentant votre plan avec des articulateurs logiques.

X Écrire le développement. Il doit y avoir un équilibre dans les parties de votre développement, c'est pourquoi vous devez prendre soin de trouver à peu près le même nombres d'arguments pour vos parties lors de votre réflexion. 
Votre développement doit être organisé. Pour cela l'utilisation de liens logiques est indispensable.

\begin{tabular}{|c|c|c|c|c|c|c|c|}
\hline \multirow{2}{*}{$\begin{array}{c}\text { Ce que je veux } \\
\text { faire ... } \\
\text { Énoncer } \\
\text { le premier } \\
\text { argument }\end{array}$} & \multicolumn{7}{|c|}{ Comment je peux le faire ... } \\
\hline & $\begin{array}{l}\text { D’abord; Tout d'abord; } \\
\text { Commençons par. }\end{array}$ & En & premier & lieu ; & Premièrement ; & Pour & $\begin{array}{c}\text { commencer } \\
;\end{array}$ \\
\hline $\begin{array}{l}\text { Ajouter un autre } \\
\text { argument }\end{array}$ & \multicolumn{7}{|c|}{$\begin{array}{l}\text { Ensuite ; De plus ; En outre ; En second lieu ; Par ailleurs ; D’autre part ; Et ; } \\
\text { Poursuivons par. }\end{array}$} \\
\hline $\begin{array}{l}\text { Énoncer } \\
\text { le dernier } \\
\text { argument }\end{array}$ & \multicolumn{7}{|c|}{ Enfin; Finalement; Pour finir; En dernier lieu. } \\
\hline $\begin{array}{l}\text { Donner un } \\
\text { exemple }\end{array}$ & \multicolumn{7}{|c|}{$\begin{array}{l}\text { C'est-à-dire ; En d'autres termes ; Par exemple ; Tel que ; Notamment ; Ainsi ; } \\
\text { Comme ; Préciser ; Illustrer ; Citer. }\end{array}$} \\
\hline $\begin{array}{r}\text { Exp } \\
\operatorname{Pr}\end{array}$ & \multicolumn{7}{|c|}{$\begin{array}{c}\text { Autrement dit ; En d'autres termes ; Quant à ; En effet ; Signifier ; Expliquer ; } \\
\text { Préciser. }\end{array}$} \\
\hline Oppc & \multicolumn{7}{|c|}{$\begin{array}{l}\text { Mais ; Pourtant ; Néanmoins ; Cependant ; En revanche ; Au contraire ; Alors que } \\
\text {; Tandis que ; Contrairement à ; Par contre ; Au lieu de ; S’opposer à ; Interdire. }\end{array}$} \\
\hline Concéder & \multicolumn{7}{|c|}{$\begin{array}{c}\text { Certes ; Toutefois ; Or ; Il est vrai ; Mis à part ; Même si ; à moins que ; Bien que ; } \\
\text { Malgré. }\end{array}$} \\
\hline $\begin{array}{l}\text { Exprimer la } \\
\text { cause }\end{array}$ & \multicolumn{7}{|c|}{$\begin{array}{c}\text { Car; Parce que ; étant donné que ; D’autant que ; à cause de ; Grâce à ; En raison } \\
\text { de ; Pour ; à la suite de ; Venir de ; Avoir pour cause ; Résulter de. }\end{array}$} \\
\hline $\begin{array}{l}\text { Exprimer la } \\
\text { conséquence }\end{array}$ & \multicolumn{7}{|c|}{$\begin{array}{c}\text { Donc; Par conséquent ; en conséquence ; C’est pourquoi ; Ainsi ; De sorte } \\
\text { que ; En sorte de ; En sorte que ; De façon que ; De manière que ; Si bien que ; } \\
\text { Pour que ; Pour ; En vue de ; Avoir pour conséquence ; Entraîner ; Provoquer ; } \\
\text { Engendrer ; Causer. }\end{array}$} \\
\hline $\begin{array}{c}\text { Séparer/Mettre } \\
\text { de côté }\end{array}$ & \multicolumn{7}{|c|}{$\begin{array}{c}\text { Ou ; Ou ... ou ; Ou bien ; Soit ... soit ; Soit que ... soit que ; D’un côté ... de l'autre ; } \\
\text { D'une part ... d'autre part ; Tantôt ... tantôt ; Si ce n'est que ; Excepté ; Sauf; Sans } \\
\text {; Hormis ; En dehors de. }\end{array}$} \\
\hline Comparer & \multicolumn{7}{|c|}{$\begin{array}{c}\text { Ainsi ; Pareillement ; Comme ; Tout comme ; De la même façon que ; Tel que ; } \\
\text { Ainsi que ; Selon ; Comparer à ; Être comparable à ; Ressembler à ; Rappeler, Se } \\
\text { rapprocher de ; Parallèlement à. }\end{array}$} \\
\hline Conclure & \multicolumn{7}{|c|}{ En conclusion ; Pour conclure ; En définitive ; En somme ; Donc. } \\
\hline
\end{tabular}


Vous pouvez donner votre opinion tout au long de l'essai ou bien dans la conclusion.

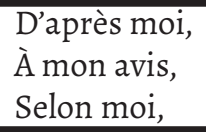

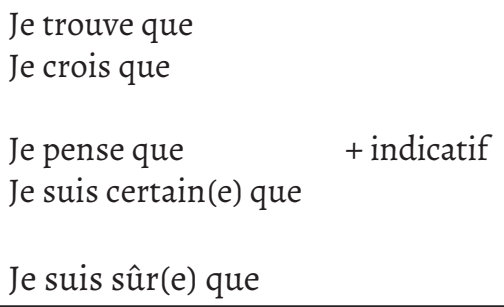

$X$ Écrire la conclusion. Tout comme l'introduction, la conclusion est très importante car ce sont les dernières phrases lues par les lecteurs. Elle est composée de trois parties.

1. Répondre à la problématique = faire une synthèse des points importants qui ont été dits sans les répéter.

2. Donner votre opinion $=$ Celle-ci peut être tranchée ou bien nuancée. Le lecteur sait que tout n'est pas tout noir ou tout blanc, il n'attend pas nécessairement une forte prise de position, mais des arguments et des exemples qui lui auront permis d'élargir sa réflexion sur le sujet.

3. Élargir le sujet $=$ une réflexion en entraîne souvent une autre, vous pouvez donc ouvrir le même sujet en le dirigeant vers une autre direction.

\section{LA RELECTURE}

$X$ Vérifier la cohérence de votre essai.

- Veillez à ce que les idées s'enchaînent de façon logique.

- Veillez à éliminer les répétions de mots, mais aussi et surtout d'idées.

- Veillez à ne pas vous contredire. 
X Éviter les clichés et les phrases toutes faites.

X Éviter la neutralité. Vous pouvez être tolérants et indiquer votre tolérance. En revanche, vous ne pouvez pas être neutre et dire : « ça m’est égal » ; «Je n’ai pas d'opinion », ...

\section{Anexo:}

Recebido para publicação em: 16 maio. 2020. Aceito para publicação em: 12 dez. 2020. 